\title{
Case Study: A BLE Communication Design of Glucose Monitor based on x73-PHD Standards and Continua Design Guidelines
}

\author{
Yuan-Fa Lee \\ Biomedical Technology and Device Research Laboratories, Industrial Technology Research Institute, Taiwan, R.O.C. \\ Email address: \\ YuanFaLee@itri.org.tw \\ To cite this article: \\ Yuan-Fa Lee. Case Study: A BLE Communication Design of Glucose Monitor based on x73-PHD Standards and Continua Design Guidelines. \\ International Journal of Wireless Communications and Mobile Computing. Vol. 4, No. 1, 2016, pp. 1-6. doi: 10.11648/j.wcmc.20160401.11
}

\begin{abstract}
The ISO/IEEE 11073 (a.k.a. x73) specifications adopted by the Continua Health Alliance constitute the international personal healthcare device (PHD) communication standards. These standards allow medical devices to intercommunicate and exchange measurement data within a single system. The Bluetooth special interest group (SIG) introduced a Bluetooth Low Energy (LE) technology with reduced power consumption. The Bluetooth LE can be used in medical devices with low data transmission rates. The research aims to identify a practical solution for the Continua BLE glucose monitor. Based on the Bluetooth LE standards as well as the Continua design guidelines and x73-PHD standards, the proposed Continua BLE glucose monitor is developed. The Continua BLE glucose monitor takes glucose measurements from the user and transmits these data to the gateway via the Bluetooth LE interface, in compliance with the Bluetooth GATT-based data format and protocol standards. Our results show that glucose measurements can be successfully transmitted to an Android-based gateway and Continua-compliant gateway through the Bluetooth LE interface. The practical solution is also feasible for other medical devices, for instance, blood pressure monitor or thermometer.
\end{abstract}

Keywords: Bluetooth LE, Continua, Glucose Monitor

\section{Introduction}

Personal healthcare systems are being aggressively marketed, and medical devices with a communication function are being widely discussed. Currently, healthcare devices have six types of transmission interface: RS-232, USB personal health device class (PHDC), Bluetooth serial port profile (SPP), Bluetooth health device profile (HDP), the ZigBee health care profile (HCP), and Bluetooth LE. In which, the transport interfaces supported by $\mathrm{x} 73$-PHD standards are USB PHDC, Bluetooth HDP, ZigBee HCP, and Bluetooth LE. Wireless transmission interfaces currently appear to be the most popular.

In 2010, we [1] proposed a novel personal healthcare system comprising a service gateway, an adapter, and a legacy device. The adapter, when combined with the legacy device, converts measured data from the legacy device, and then transmits these data to the service gateway via a Bluetooth interface. The adapter communicates with the legacy device via a universal asynchronous receiver/transmitter (UART) interface. In 2012, Park and his colleagues [2] presented an implementation model of standardization for legacy healthcare devices. The proposed system generates standard PHD protocol message blocks using legacy device information via a UART interface.

In 2013, we [3] introduced a personal medical monitoring system based on the international x73-PHD standards [4]. The proposed solution, a two-in-one blood pressure plus blood glucose monitoring system, enables wireless transmission functionality by adopting the IEEE 802.15.1 Bluetooth standard and a new HDP [5]. In 2015, Jiho Kim and colleagues [6] proposed an ISO/IEEE 11073 standardization system, including a ZigBee adaptation module (ZAM), and a PHD manager. The ZAM communicates with a healthcare device through a UART interface and transmits measurement data to the PHD manager via the ZigBee interface. These studies proposed $\mathrm{x} 73$-PHD solutions but focused on the Bluetooth HDP or ZigBee HCP interface.

In 2013, Park and colleagues [7] proposed a patch-type ECG sensor system (ECG node) and an android application. The ECG sensor node makes a packet which is transmitted to the smart device after sensing ECG data. 
In 2014, Lin and colleagues [8] proposed a Bluetooth low energy based blood pressure monitoring system, which integrated a homemade blood pressure measuring device and a smartphone. The device uses single BLE System-on-Chip to process the algorithm of blood pressure measurement and data transmission. However, these solutions didn't adopt x73-PHD standardizations and Continua design guidelines. They didn't consider the interoperability of medical devices. Thus, different types of medical devices can't all communicate with the same gateway or application host device (AHD).

In this study, we propose a Continua BLE glucose monitor that follows the Bluetooth LE standards and Continua design guidelines v4.0 [9-12]. This system converts the communication protocol of legacy devices to the Bluetooth LE protocols [10-12]. In addition, an interoperability design of the $\mathrm{x} 73-\mathrm{PHD}$ standardizations is developed. It is further integrated into the Bluetooth smart ready gateway, which is also an IEEE 11073 PHD compatible manager.

Our research aims to identify a practical solution for the Continua BLE glucose monitor. The proposed method including System Architecture, Use Case Analysis, Message Flow Sequence, and GATT Profile Design is introduced in the Methods section of this paper. Our test results from the lab work are presented in the Results and Discussion section, and our concluding remarks are given in the Conclusions section.

\section{Methods}

\subsection{System Architecture}

The Continua BLE glucose monitor consists of a Continua BLE system and a glucose sensor system. The Continua BLE system connects with a gateway via the Bluetooth GATT-based interface and connects with the glucose sensor system via a wired UART interface. The measurement data are exchanged between the Continua BLE glucose monitor and the gateway. Figure 1a gives an operational overview of the proposed Continua BLE glucose monitor. It offers an interoperability platform for measurement data exchange that is compliant with the international Bluetooth LE and x73-PHD standards via a standard low power (LP) wireless PAN interface.

Figure $1 \mathrm{~b}$ illustrates the software system architecture of the Continua BLE glucose monitor based on a Texas Instruments BLE CC2540/41 SDK (Software Development Kit) [13]. The Continua BLE glucose monitor consists of several modules including a link layer, the logical link control and adaptation protocol (L2CAP), the generic access profile (GAP), the generic attribute profile (GATT), a security manager, the GAP role, the GAP bond manager, a glucose profile/service, and an uart_if. The uart_if module is designed to communicate with the glucose sensor system.

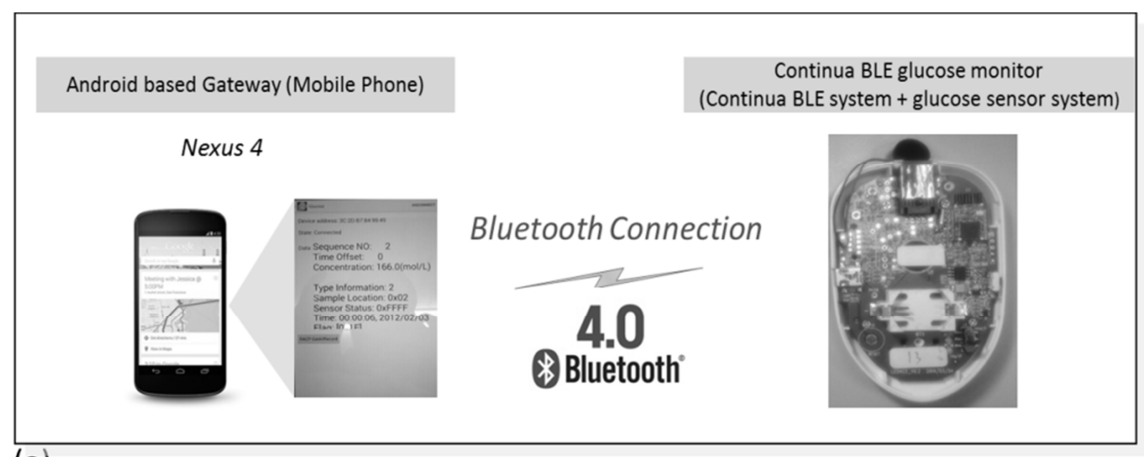

(a)

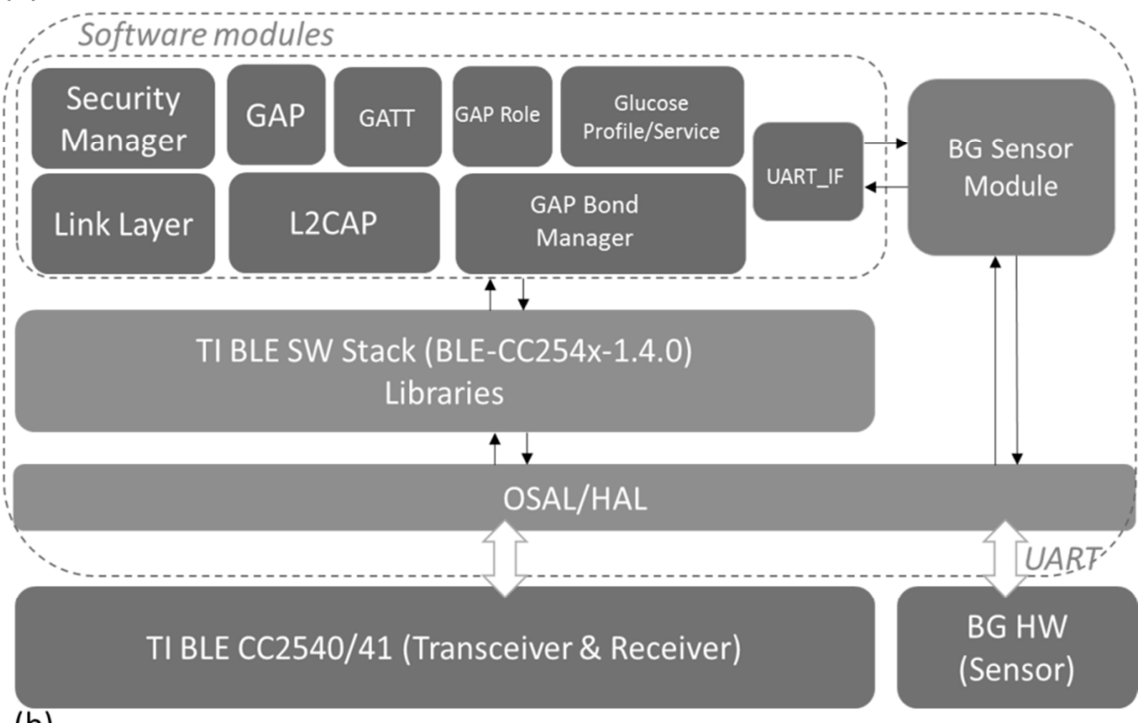

(b)

Fig. 1. (a) A operational overview of the Continua BLE glucose monitor connected to an Android-based mobile phone via a Bluetooth LE interface. (b) A system architecture overview of the Continua Bluetooth LE glucose monitor. 


\subsection{Use Case Analysis}

The proposed Continua BLE glucose monitor consists of a Continua BLE system and a glucose sensor system in which the Continua BLE system adopts the iMCC2541 module (TI BLE CC2540/41 based) as the Bluetooth LE solution. The glucose sensor is supported by Wisdom IOT-Biomedical Technology Inc. The Continua BLE glucose monitor uses the wireless transmission of Bluetooth LE and meets the x73-PHD standards. Figure 2a illustrates the use case analysis.

Taking the Continua BLE system as the kernel, there are six main functions: start system, stop system, send advertisement, get vital sign information, establish BLE data channel, and send out BLE data. The glucose sensor system provides power to the Continua BLE system at system start and cuts off the power at system stop. An advertisement message is periodically sent out by the Continua BLE system when the RF button is pressed. At the same time, the Continua BLE glucose system becomes discoverable to the GATT client (i.e., gateway). A Continua-compliant gateway connects with the Continua BLE system. The Continua BLE system acquires measurement data from the glucose sensor system and sends them to the gateway after parsing and encapsulating the data in Bluetooth LE message format.

\subsection{Message Flow Sequence}

Figure $2 \mathrm{~b}$ illustrates the overall message flow sequence of the system components: gateway, Continua BLE system, and glucose sensor system. After the Continua BLE system wakes up from sleep mode, it starts to send out advertising messages if system initialization is successful. A gateway attempts to connect with the Continua BLE system by sending a connect request message. If the Continua BLE system accepts the connection request, the devices start to exchange information. The Continua BLE system notifies the glucose sensor system that a Bluetooth LE data channel has been established by sending an online test message. The glucose sensor system responds to a successful message with online ready. The Continua BLE system starts to read measured data from the glucose sensor system and sends the data, with an indication or notification procedure to the gateway. The Continua BLE system notifies the glucose sensor system that the measurement data have been transmitted successfully by sending an upload complete message, and the glucose sensor system labels the measurement data as transmitted. The Continua BLE system reads the measurement data sequentially and sends them out to the gateway until all measurement data have been transmitted successfully. The Continua BLE system disconnects the Bluetooth LE connection automatically and enters the sleep mode again.

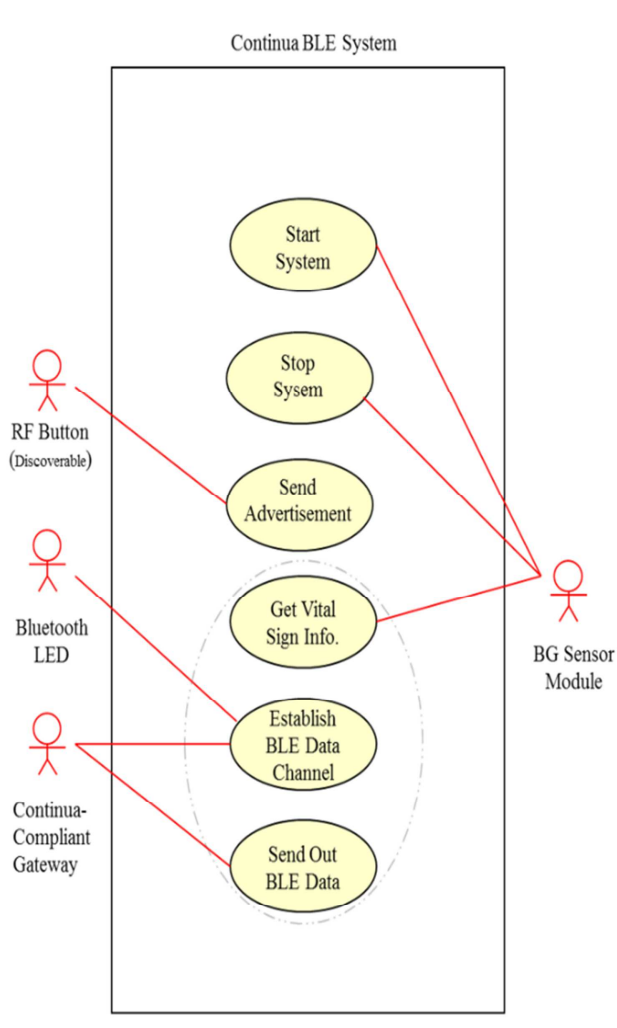

(a)
Continua BTLE glucose monitor

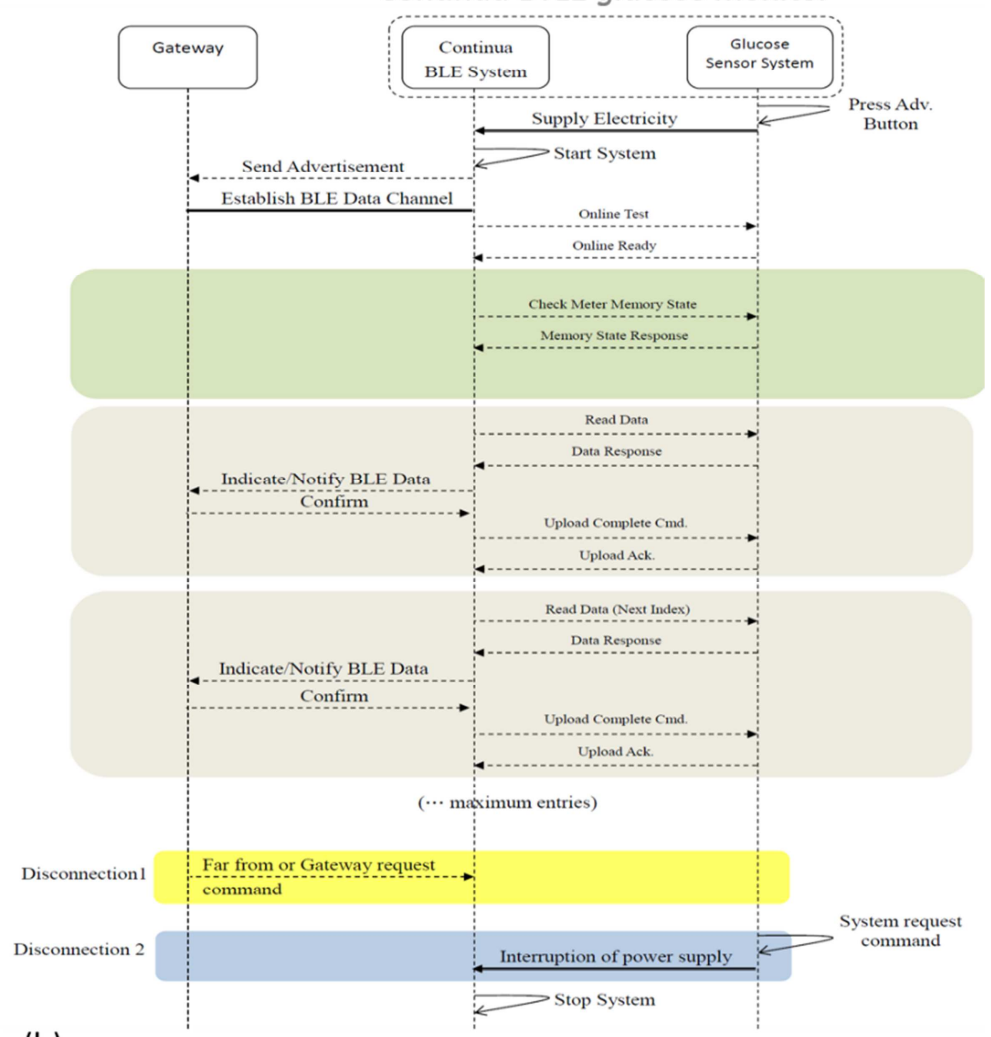

(b)

Fig. 2. (a) Analysis of the Continua BLE system use case. (b) A work flow overview of the Continua BLE system with a glucose sensor system conjoined for connecting to the gateway. 


\subsection{GATT Profile Design}

Figure 3 illustrates the GATT profile design of the Continua BLE glucose monitor. The GATT profile is designed to be used by an application, allowing the client to communicate with a server. The server has a number of attributes, and the GATT profile defines how to use the attribute protocol to discover, read, write, and obtain other details of these attributes, as well as configuring the broadcast of the attributes.

Based on the Bluetooth glucose protocol and service specifications [11, 12], the GATT profile of the Continua BLE glucose monitor has three primary services: the GAP service (whose universally unique identifier (UUID) is $0 x 1800$ ), the glucose service (whose UUID is 0x1808), and the device information service (whose UUID is 0x180A). The GAP service has two characteristics: device name and appearance.
The glucose service has four characteristics: glucose measurement, glucose feature, record access control point, and date time. The device information service has six characteristics: system identity, model name, manufacturer's name, firmware revision, serial number, and IEEE 11073-20601 regulatory certification data list [4]. The Continua BLE glucose monitor supports a GATT client to access these attributes using ATT commands, including discovery service, read a characteristic value, write a characteristic value, indication of a characteristic value and notification of a characteristic value [10].

In addition, the date time characteristic of the glucose service and the IEEE 11073-20601 regulatory certification data list of the device information service are added to the GATT profile to meet the requirements of the Continua design guidelines v4.0.

\begin{tabular}{|c|c|c|c|c|}
\hline ConHnd & Handle & Uuid & Uuid Description & Value \\
\hline $0 \times 0000$ & $0 \times 0001$ & $0 \times 2800$ & GATT Primary Service Declaration & $00: 18$ \\
\hline $0 \times 0000$ & $0 \times 0002$ & $0 \times 2803$ & GATT Characteristic Declaration & $02: 03: 00: 00: 2 \mathrm{~A}$ \\
\hline $0 \times 0000$ & $0 \times 0003$ & $0 \times 2 A 00$ & Device Name & $42: 47: 20: 4 D: 65: 74: 65: 72$ \\
\hline $0 \times 0000$ & $0 \times 0004$ & $0 \times 2803$ & GATT Characteristic Declaration & $02: 05: 00: 01: 2 A$ \\
\hline $0 \times 0000$ & Cx0005 & $0 \times 2 A 01$ & Appearance & $00: 00$ \\
\hline $0 \times 0000$ & Ox0006 & $0 \times 2800$ & GATT Primary Service Declaration & 08:18 \\
\hline $0 \times 0000$ & $0 \times 0007$ & $0 \times 2803$ & GATT Characteristic Declaration & $10: 08: 00: 18: 2 A$ \\
\hline $0 \times 0000$ & Ox0008 & O $\times 2 A 18$ & Glucose Measurement & \\
\hline $0 \times 0000$ & $0 \times 0009$ & $0 \times 2902$ & Client Characteristic Configuration & \\
\hline $0 \times 0000$ & $0 \times 000 \mathrm{~A}$ & $0 \times 2803$ & GATT Characteristic Declaration & $02: 0 B: 00: 51: 2 A$ \\
\hline $0 \times 0000$ & $\mathrm{O} \times 000 \mathrm{~B}$ & $0 \times 2 A 51$ & Glucose Feature & $00: 00$ \\
\hline $0 \times 0000$ & $0 \times 000 \mathrm{C}$ & $0 \times 2803$ & GATT Characteristic Declaration & $28: 0 D: 00: 52: 2 A$ \\
\hline $0 \times 0000$ & Qx000D & Cx2A52 & Record Access Control Point & \\
\hline $0 \times 0000$ & OXOOOE & $0 \times 2902$ & Client Characteristic Configuration & \\
\hline $0 \times 0000$ & Ox000F & $0 \times 2803$ & GATT Characteristic Declaration & $02: 10: 00: 08: 2 A$ \\
\hline $0 \times 0000$ & $0 \times 0010$ & $0 \times 2 A 08$ & Date Time & DD:07:0A:1F:0E:0C:00 \\
\hline $0 \times 0000$ & Ox0011 & $0 \times 2800$ & GATT Primary Service Declaration & $\mathrm{OA}: 18$ \\
\hline $0 \times 0000$ & $0 \times 0012$ & $0 \times 2803$ & GATT Characteristic Declaration & $02: 13: 00: 23: 2 A$ \\
\hline $0 \times 0000$ & $0 \times 0013$ & $0 \times 2 A 23$ & System ID & $00: 00: 00: 00: 00: 00: 00: 00$ \\
\hline $0 \times 0000$ & Ox0014 & $0 \times 2803$ & GATT Characteristic Declaration & $02: 15: 00: 24: 2 A$ \\
\hline $0 \times 0000$ & Ox0015 & $0 \times 2 A 24$ & Model Number String & $54: 44: 33: 32: 36: 31: 46$ \\
\hline $0 \times 0000$ & Ox0016 & $0 \times 2803$ & GATT Characteristic Declaration & $02: 17: 00: 29: 2 A$ \\
\hline $0 \times 0000$ & Cx0017 & $0 \times 2 A 29$ & Manufacturer Name String & $57: 2 D: 69: 4 F: 54: 20: 43: 6 F: 2 E$ \\
\hline $0 \times 0000$ & Ox0018 & $0 \times 2803$ & GATT Characteristic Declaration & $02: 19: 00: 26: 2 A$ \\
\hline $0 \times 0000$ & $0 \times 0019$ & $0 \times 2 A 26$ & Fimware Revision String & $31: 2 \mathrm{E}: 30: 30: 2 \mathrm{D}: 42: 30: 31$ \\
\hline $0 \times 0000$ & Ox001A & $0 \times 2803$ & GATT Characteristic Declaration & $02: 1 \mathrm{~B}: 00: 25: 2 \mathrm{~A}$ \\
\hline $0 \times 0000$ & Cx001B & $0 \times 2 A 25$ & Serial Number String & $30: 30: 30: 30: 30: 30: 30: 30: 30: 30$ \\
\hline $0 \times 0000$ & Ox001C & $0 \times 2803$ & GATT Characteristic Declaration & $02: 1 \mathrm{D}: 00: 2 \mathrm{~A}: 2 \mathrm{~A}$ \\
\hline $0 \times 0000$ & Cx001D & $\mathrm{O} \times 2 \mathrm{~A} 2 \mathrm{~A}$ & IEEE 11073-20601 Regulatory Certificati... & $00: 02: 00: 12: 02: 01: 00: 08: 02: 00: 00: 01: 00: 02: 11: 80: 02: 02: 00: 02: 00: 00$ \\
\hline
\end{tabular}

Fig. 3. The GATT profile design for the Continua BLE glucose monitor.

\section{Results and Discussion}

The system proposed in this study was developed based on a previous implementation [1, 3]. The Android based gateway was developed on a Google Nexus 4 mobile phone, while the Continua AHD was developed and integrated with CESL [14] using a low power wireless PAN-IF solution on a Windows-based platform. The Continua BLE system was developed in an embedded system with RTOS support and used protocol and device specifications defined in the Bluetooth GATT based standards [10-12]. The Continua BLE system utilized MCU (TI BLE CC2541) as its micro-controller unit. It communicated with the glucose sensor system through the UART interface. The Continua BLE glucose monitor was tested using Continua Test Management Lite, version 4.0.0.0, and Continua CESL Manager, version 4.0 [15]. For the duration of the test process the air traffic between the Continua BLE glucose monitor and the gateway/Continua AHD was sniffed by a Frontline Bluetooth protocol analyzer (BPA). The captured traffic was used for diagnosis and troubleshooting of problems. All test results were positive. Table 1 shows the laboratory equipment. 
Table 1. Laboratory equipment.

\begin{tabular}{|c|c|c|}
\hline \multirow{2}{*}{ Device Name } & \multicolumn{2}{|c|}{ Personal Healthcare System } \\
\hline & Item & Used in the test \\
\hline \multirow{2}{*}{$\begin{array}{l}\text { Android-based } \\
\text { Gateway }\end{array}$} & & Google Nexus 4 (Phone) \\
\hline & Hardware & *Support Bluetooth LE Feature (as \\
\hline \multirow{3}{*}{ Continua AHD } & Hardware/ & 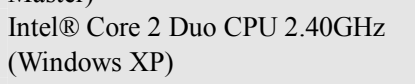 \\
\hline & & CSR 8510 USB dongle (as Master) \\
\hline & Software & $\begin{array}{l}\text { CESL Manager \& Test Management } \\
\text { Lite }\end{array}$ \\
\hline $\begin{array}{l}\text { Continua BLE } \\
\text { Glucose Monitor }\end{array}$ & Hardware & iMCC2541 module (as Slave) \\
\hline TI CC2540/41 & & TI SmartRF05 Evaluation Board \\
\hline $\begin{array}{l}\text { BLE Evaluation } \\
\text { Board }\end{array}$ & Hardware & $\begin{array}{l}\text { CC2540 Evaluation Module (with } \\
\text { BLE-CC254x-1.4.0) }\end{array}$ \\
\hline Frontline BPA & Hardware & Bluetooth Protocol Analyzer \\
\hline
\end{tabular}

When the RF button was pressed the Continua BLE glucose monitor started periodically to send an advertising message.
The gateway connected with the Continua BLE glucose monitor by sending a connect request message, and a physical Bluetooth channel was successfully created. The gateway started to interrogate the GATT profile of the Continua BLE glucose monitor with ATT request commands, to which the monitor responded with corresponding ATT response commands. The transaction continued until the gateway had captured the GATT profile of the Continua BLE glucose monitor. A GATT based data channel was concurrently created. The Continua BLE glucose monitor then started to transmit its measured data to the gateway.

Because of the low power consumption and limited message transmission of Bluetooth LE, the system operation of the Continua BLE glucose monitor was divided into three phases: advertisement, GATT profile interview, and measurement data transmission.

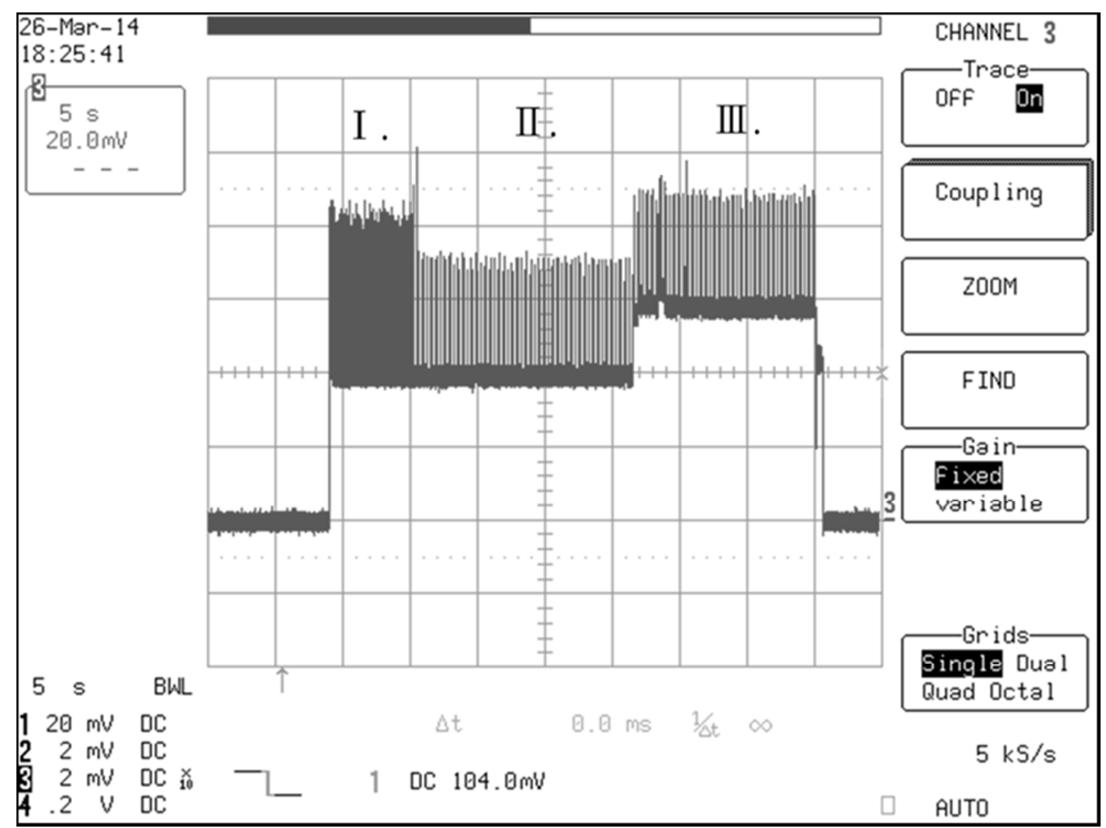

Fig. 4. The three phases of current consumption for the Continua Bluetooth LE glucose monitor: advertising, interrogating GATT profile, and transmitting measurement data.

\subsection{Advertisement}

The Bluetooth specifications $[11,12]$ recommend that the GAP peripheral role advertising follows two time durations: fast connections (first 30 seconds) and slow connections (after 30 seconds). For fast connections, the advertising interval is 20 to $30 \mathrm{~ms}$. For slow connections, the advertising interval is 1 to $2.5 \mathrm{~s}$. The interval values in the first connection are designed to attempt fast connection during the first $30 \mathrm{~s}$; however, if a connection is not established within that time, the interval values in the second connection are designed to the reduce power consumption of devices that continue to advertise. It is also recommended that the GAP central role use the recommended scan interval and scan window values. For the first $30 \mathrm{~s}$, the GAP central should use the first scan window $(30 \mathrm{~ms})$ and scan interval (30 to $60 \mathrm{~ms}$ ) pair to attempt fast connection. However, if a connection is not established within that time, the GAP central should switch to one of the other scan window/scan interval options to reduce power consumption. The Continua BLE glucose monitor only advertises itself in the first 30s. After that, the monitor will stop advertising and enter the stop system phase. It will enter the advertisement phase and start sending advertisement message only if the user presses the RF button again. The current design offers low power consumption in the advertisement phase.

\subsection{GATT Profile Interview}

GATT profiles vary according to the type of medical device used and the product features. Some BLE medical devices need to include more services in their GATT profiles, and some need to add or subtract service characteristics. The interview time and the current consumption vary according to 
the size of the GATT profile. To reduce the interview time of a GATT profile, the GATT client currently supports a cache mechanism to remember the profile of the last BLE medical device that was connected. The GATT profile is interrogated only at the first time of connection. The interview time of the profile at the next connection is ignored, and the Continua BLE glucose monitor enters the measurement data transmission phase directly. The total connection time is shortened and total power consumption is reduced. Our Android-based gateway supported the cache mechanism, allowing the interview time of the GATT profile to be ignored. However, the test tools (Continua CESL manager and Continue test management lit) did need to interview the GATT profile at every connection time because they need to verify the interoperability and functionalities of the BLE medical device being tested.

\subsection{Measurement Data Transmission}

Depending on the Bluetooth specification [10], the BLE measurement data has a fixed message format and size. The criterion for power consumption in this phase is that the same measured data is transmitted repeatedly. For example, the use case of some BLE medical devices is that measurement data is not be erased and can be transmitted repeatedly. The total transmission time is then higher, and the power consumption is also higher. The Continua BLE glucose monitor has a limited maximum volume of measurement data. The measurement data is labeled as transmitted if it is transmitted successfully. The transmitted measurement data is not transmitted again and can be erased by the user, or automatically by the system. Therefore, the total transmission time is lowest and the power consumption is lowest for the Continua BLE glucose monitor.

Based on our implementation and experience, we found the Bluetooth related specifications [10-12] cover the design requirement of GATT profile almost completely. In addition, based on Continua design guidelines v4.0, only two characteristics (date time and IEEE 11073-20601 regulatory certification data list) need to be added to the GATT profile to meet Continua product requirements. These are that the date time characteristic should be added to the glucose service of the GATT profile, and that the IEEE 11073-20601 regulatory certification data list characteristic should be added to the device information service of the GATT profile. The Continua BLE glucose monitor meets both the requirements of the Bluetooth LE specifications and the Continua low power wireless PAN specifications.

\section{Conclusions}

This study introduced a Bluetooth LE based Continua glucose monitor. We have demonstrated that the Continua BLE glucose monitor is capable of connecting to and exchanging measurement data with an Android-based mobile phone with Bluetooth smart ready capability. The Continua BLE glucose monitor offers an interoperable platform for personal healthcare ecosystems based on the ISO/IEEE 11073 transport-independent personal-health data and protocol standards with low power wireless PAN-IF features.

In the experiment, we created a Continua BLE system based on TI BLE CC2540/41 chipset. This standardization system can be adapted to many legacy healthcare devices. We demonstrated that the system offers a practical approach to constructing an e-health service environment based on the international $\times 73-P H D$ standards and Bluetooth LE technology. Future research will focus on supporting other types of healthcare devices and other kinds of device specifications, for example ECG and cardiovascular, and fitness devices.

\section{References}

[1] Y.-F., Lee and Y.-S. Huang, "Novel Personal Healthcare System," $4^{\text {th }}$ Intl. Symposium on Medical Info. and Communication Technology, 2010.

[2] C.-Y. Park, J.-H. Lim, and S.-J. Park, "ISO/IEEE 11073 PHD Adapter Board for Standardization of Legacy Healthcare Device,” IEEE Intl. Conf. on Consumer Electronics, 2012.

[3] Y.-F. Lee, "Personal Medical Monitoring System Based on x73-PHD Standards," IEEE IT Professional, vol. PP, Issue 99, September 2012.

[4] IEEE, Health Informatics - Personal Health Device Communication. Part 20601: Application Profile - Optimized Exchange Protocol, 2010.

[5] Bluetooth SIG, Health Device Profile, 2008.

[6] J. Kim and O. Song, "ISO/IEEE 11073 interoperability for person health device based on ZigBee healthcare service," IEEE Intl. Conf. on Consumer Electronics (ICCE), pp. 263-264, 2015.

[7] Y.-J. Park, and H.-S. Cho, "Transmission of ECG Data with the Patch-Type ECG Sensor System using Bluetooth Low Energy," Intl. Conf. on ICT Convergence (ICTC), pp. 289-294, 2013.

[8] Z.-M. Lin, C.-H. Chang, N.-K. Chou, and Y.-H. Lin, "Bluetooth Low Energy (BLE) Based Blood Pressure Monitoring System," Intl. Conf. on Intelligent Green Building and Smart Grid (IGBSG), pp. 1-4, 2014.

[9] Continua Health Alliance, Continua Design Guidelines Version 4.0, 2013.

[10] Bluetooth SIG, Bluetooth Specification Version 4.0, 2010.

[11] Bluetooth SIG, Glucose Service, 2012.

[12] Bluetooth SIG, Glucose Profile, 2012.

[13] (2015, July.). Bluetooth low energy software stack and tools. [Online]. http://www.ti.com/product/CC2540/toolssoftware

Available:

[14] (2015, July.). Continua Enabling Software Library (CESL). [Online].

Available: https://cw.continuaalliance.org/wg/members/home/cesl-downl oad.

[15] (2015, July.). Continua Test Tool. [Online]. Available: https://cw.continuaalliance.org/wg/members/home/test-tool-do wnload. 